\title{
Predicting Malignancy in FDG-avid Thyroid Nodules based on Standardized Uptake Value in Oncology Patients
}

\author{
Michael Bozin ${ }^{1}$, Jason Callahan ${ }^{2}$, Elizabeth Drummond ${ }^{3}$, Michael Henderson $^{4}$, Anita Skandarajah ${ }^{5}$
}

\begin{abstract}
Aim and objective: Concomitant thyroid nodules and cancer found incidentally on 18-fluorodeoxyglucose (FDG) positron-emission tomography (PET-CT) in patients imaged for investigation or staging of malignancy have been progressively encountered. Maximum SUV (SUV-max) may aid diagnosis of concomitant thyroid malignancy.

Materials and methods: PET/CT scans were reviewed between 2009 and 2014 in which FDG-avid thyroid incidentalomas were included. Patients who were investigated with biopsy or surgery were further assessed to determine the utility of SUV-max, mean, and nodule size as a determinate of malignancy.

Results: A total of 325 of 35,586 patients were identified with FDG-avid thyroid incidentalomas (TI)-99 were investigated. The incidence was $0.88 \%$. Forty nine were malignant, with a median SUV-max of 14.5 (range 2.7-60.4). Malignant nodules had higher median SUV-max than benign nodules at a threshold of $5(p<0.0001)$. Receiver operator curve (ROC) analysis demonstrated an area under the curve of $0.66(95 \% \mathrm{Cl} 0.55-0.77$, $p=0.005)$. The sensitivity and specificity were 73.4 and 46.9 , respectively. The positive and negative likelihood ratio was $3.12(95 \% \mathrm{Cl} 1.80-5.50)$ and a negative likelihood ratio of 0.38 .

Conclusion: As FDG-avid TI can demonstrate variable uptake, SUV-max is not a useful tool to discriminate benign from malignant TI. SUV-max should remain a theoretical adjunctive tool for predicting thyroid cancer.

Clinical significance: The threshold for investigating PET-avid thyroid nodules with ultrasound and FNAC (in the context of primary nonthyroidal malignancy) should be determined by the prognosis of the primary malignancy.

Keywords: Cohort study, Positron-emission tomography, Thyroid cancer, Thyroid nodule.

World Journal of Endocrine Surgery (2021): 10.5005/jp-journals-10002-1409
\end{abstract}

\section{INTRODUCTION}

Fluorine-18 fluorodeoxyglucose (FDG) positron-emission tomography/computed tomography (PET/CT) is an accurate functional imaging technique increasingly used for the diagnosis, staging, and therapeutic monitoring of many common cancers. PET detected or "avid" "incidentalomas" have been reported to occur in up to $5 \%$ of scans. Incidentally detected thyroid nodules on FDG-PET/CT have been reported to occur in up to $2.5 \%$ of scans. ${ }^{1}$ The incidence of these "thyroid incidentalomas" (TI) varies widely within the literature, with a range from $0.02-8.9 \%$ from studies ranging from $689-15,711$ patients. ${ }^{1-3}$ Rates of malignancy varying also widely between $13-59 \%$ of $\mathrm{TI}^{2}$

Cancer risk stratification of thyroid nodules and incidentalomas utilizing the maximum standardized uptake value (SUV-max) of 18-FDG as a surrogate marker of malignancy has also been proposed within the literature. ${ }^{4,5}$ Generally, cancerous lesions will have a higher SUV-max than their benign counterparts due to their inherent abnormalities in aerobic glycolysis. A study by Bertagna et al. observing the utility of SUV-max of 4.8 to predict thyroid cancer yielded a sensitivity and specificity of 95.7 and $46.4 \%$, respectively. ${ }^{1}$ However, benign nodules such as Hürthle cell and follicular adenomas may also have high SUV-max, which act as confounders. ${ }^{6}$ Therefore, controversy remains surrounding the utility of SUV-max as there can be considerate overlap between benign and malignant lesions., ${ }^{7,8}$

The primary outcome of this study is to determine if maximum standardized uptake value (SUV-max), representing the maximum uptake of 18-FDG, can be used as a surrogate measure of

\begin{abstract}
${ }^{1}$ Department of Specialist General Surgery, Royal Melbourne Hospital, Melbourne, Victoria, Australia

2,3 Department of Cancer Imaging, Peter MacCallum Cancer Centre, Melbourne, Victoria, Australia

${ }^{4,5}$ Department of Cancer Surgery, Peter MacCallum Cancer Centre, Melbourne, Victoria, Australia

CorrespondingAuthor:MichaelBozin,DepartmentofSpecialistGeneral Surgery, Royal Melbourne Hospital, Melbourne, Victoria, Australia, Phone: +613 8559 5000, e-mail: mikebozin@gmail.com

How to cite this article: Bozin M, Callahan J, Drummond E, et al. Predicting Malignancy in FDG-avid Thyroid Nodules based on Standardized Uptake Value in Oncology Patients. World J Endoc Surg 2021;13(2):42-46.
\end{abstract}

Source of support: Nil

Conflict of interest: None

malignancy risk in cancer patients with thyroid nodules incidentally detected on PET/CT.

\section{Materials and Methods}

A retrospective review was performed of patient demographics, imaging (PET/CT scans and reports), and pathology in patients with a primary cancer diagnosis and incidentally detected thyroid nodules on PET/CT. This was performed on patients who underwent a PET/CT at a single tertiary level cancer center between January 2007 to January 2015 for the purpose of diagnosis, staging, or treatment response in relation to a patient's primary malignancy.

() The Author(s). 2021 Open Access This article is distributed under the terms of the Creative Commons Attribution 4.0 International License (https://creativecommons. org/licenses/by-nc/4.0/), which permits unrestricted use, distribution, and non-commercial reproduction in any medium, provided you give appropriate credit to the original author(s) and the source, provide a link to the Creative Commons license, and indicate if changes were made. The Creative Commons Public Domain Dedication waiver (http://creativecommons.org/publicdomain/zero/1.0/) applies to the data made available in this article, unless otherwise stated. 
Ethics approval was obtained from the Department of Surgical Oncology ethics review board (number 17/17R).

All PET/CT scans were performed with Discovery 390, Biograph, and Discovery STE PET/CT scanner systems (GE Medical Systems, Waukesha, WI, USA). PET/CT scan reports were reviewed using "thyroid" as a search term. Patient duplicates and scans with normal thyroid reports were excluded. Other exclusions (non-FDG-avid incidentalomas) included diffuse or physiological uptake to the thyroid gland, thyroiditis, patients known thyroid cancer, reported multinodular goiter without FDG-avidity, nodules without FDG-activity, focal uptake less than $1 \mathrm{~mm}$ without association with nodule on CT and nodules identified with non-FDG radiotracers (GA-Tate, 1-Flurocholine, PSMA) (Fig. 1).

Patients who were investigated for their thyroid incidentalomas by FNAC or surgery were further assessed to determine the utility of SUV-max as a determinate of malignancy. Fine needle aspirate results were document in accordance with the Bethesda classification of reporting thyroid nodule cytopathology. ${ }^{9}$ Histopathology results/thyroid pathology were documented in accordance with the WHO classification of endocrine tumors. ${ }^{10}$

FDG-avidity parameter data was gathered using MIM imaging software (MIM, OH, USA). This was conducted by identifying the thyroid nodule on a maximum projection image (MIP) and selecting PET EDGE tool to delineate the thyroid nodule. Dimensions of the selected area of avidity was recorded using the RECIST tool. The process was repeated using a homogenous area of the liver to gather quality assurance data related to uptake of FDG (aiming for a SUV-mean between 2 and 3). Only the index PET/CT scan was assessed. This was a single time-point cross-sectional analysis that was defined as the patients PET/CT scan that first identified the nodule and/or was the scan immediately prior to investigation with FNAC or surgery. FDG-avidity parameters recorded included SUV-max, SUV-mean, dimensions of the thyroid nodule, SUV-mean of the liver as quality assurance, and dose of FDG and uptake time.

Fisher's exact test was used to determine if SUV-max was significant for malignant nodules at a given uptake. An SUV-max threshold of 5 was used as per Bertagna et al.

A receiver operating characteristic $(\mathrm{ROC})$ curve analysis of the patients with a definitive diagnosis of thyroid malignancy was performed to determine the diagnostic performance of the test

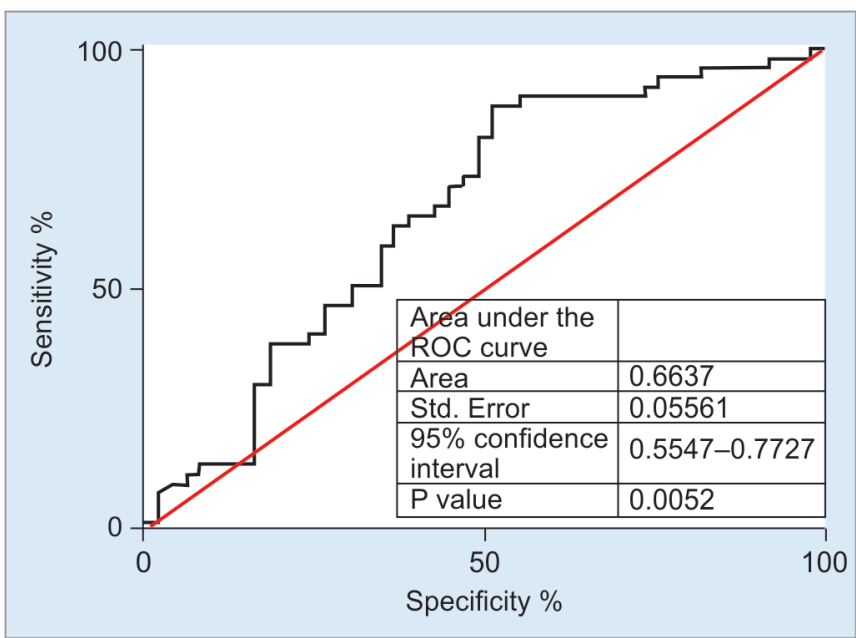

Fig. 1: Receiver operating characteristic (ROC) curve demonstrating performance of SUV-max predicting thyroid malignancy and identify a SUV cutoff useful in differentiating benign from malignant incidentalomas.

Linear regression analysis with Pearson correlation was also performed to determine an association between SUV-max and nodular diameter $(\mathrm{mm})$.

\section{Results}

PET/CT scan reports of 36,596 consecutive patients from PET/CT database were reviewed, identifying 2,588 FDG PET/CT reports referring to the thyroid. Patient duplicate scans were excluded revealing 1,618 patients with thyroid abnormalities. Non-FDG-avid incidentalomas were excluded, identifying 325 patients with FDG-avid TI. Ninety-nine patients were investigated had cytological or histological proven benign or malignant thyroid nodules with a median age of 72 (range 26-97). The most common primary malignancy was lymphoma amongst 15 patients, followed by colorectal cancer in nine patients, lung cancer in 8 patients, and melanoma in eight patients (Table 1). The incidence of FDG-avid TI was therefore found to be $0.88 \%$.

Forty-nine patients were found to have malignant thyroid nodules, with a median SUV-max of 14.5 (range 2.7-60.4) and SUV-mean of 9.3. The incidence of malignancy amongst all FDG-avid $\mathrm{TI}$ and investigated nodules was $15 \%$ and $49.5 \%$, respectively (Table 2). Histological diagnosis demonstrated 28 papillary thyroid cancers, eight were metastasis to the thyroid, three follicular thyroid cancers, two Hürthle cell cancers, and one medullary thyroid cancer. Seven patients did not proceed to core biopsy or surgery but were found to have malignant nodules on FNAC (Table 3).

Fifty patients were found to have benign thyroid nodules, with a median SUV-max of 8.6 (range 1.9-48.2), and a SUV-mean of 6.4. Mean diameter $(\mathrm{mm})$ of both malignant and benign nodules was 18 (Table 2).

Fisher's exact test confirmed that malignant nodules had higher median SUV-max than benign nodules at a SUV-max threshold of 5, with a $p$-value of less than 0.0001 . The sensitivity and specificity of SUV-max at this threshold were $70.9 \%(95 \% \mathrm{Cl} 57.1-82.4)$ and $77.2 \%$ (95\% Cl 62.2-88.5), respectively. This corresponded to a positive likelihood ratio of $3.12(95 \% \mathrm{Cl} 1.80-5.50)$ and a negative likelihood ratio of 0.38 ( $95 \% \mathrm{Cl} 0.24-0.59)$.

Receiver operating characteristic curve analysis was performed to determine the diagnostic performance of SUV-max. The SUV-max threshold which was found to give the best possible separation of benign and malignant TI was 5.33. This demonstrated

Table 1: Demographics of investigated cancer patients with FDG-avid TI

Patient demographics

Age (years)

Median

Range

72

Gender (N)

Male 26-97

Female

Primary malignancy, N (\%)

Lymphoma

Lung

$8(8)$

Colorectal

9(9)

Melanoma

8(8)

Other

59(60) 
Table 2: FDG-avid thyroid nodule characteristics based on SUV-max and size

\begin{tabular}{|c|c|c|c|c|c|c|c|}
\hline & $N$ & $S U V<5(N)$ & $S U V>5(N)$ & Median SUV max & $S D$ & $\begin{array}{l}\text { SUV } \\
\text { mean }\end{array}$ & Size (mm) \\
\hline Malignant & 49 & 10 & 39 & $\begin{array}{c}14.5 \\
(2.7-60.4)\end{array}$ & 12 & 9.3 & 18 \\
\hline Benign & 50 & 34 & 16 & $\begin{array}{c}8.6 \\
(1.9-48.2)\end{array}$ & 8.2 & 6.4 & 18 \\
\hline
\end{tabular}

Table 3: Histopathological and cytological assessment of FDG-avid thyroid nodules

\begin{tabular}{lcc}
\hline $\begin{array}{l}\text { Nodule type on FNAC } \\
\text { or histopathology }\end{array}$ & $N(\%)$ & Median SUV-max \\
\hline Benign & $50(51)$ & 8.6 \\
Malignant & $49(49)$ & 14.5 \\
Papillary & $28(28)$ & 8.8 \\
Follicular & $3(3)$ & 17.1 \\
Medullary & $1(1)$ & 6.1 \\
Hürthle cell & $2(2)$ & 15.3 \\
Metastasis & $8(8)$ & 7 \\
Malignant on FNA & $7(7)$ & 11.1 \\
alone & & \\
\hline
\end{tabular}

an area under the ROC curve of $0.66(95 \% \mathrm{Cl} 0.55-0.77)$, with a $p$-value of 0.005 (Fig. 1). This threshold demonstrated a sensitivity and specificity of 73.4 and 46.9 , respectively. Linear regression demonstrated no correlation between SUV-max and nodular size (R-square 0.004, $p$-value 0.52).

\section{Discussion}

Thyroid cancer incidence is rising at a faster rate than any other solid tumor in the United States of America (USA), as shown between 1975 and 2009, where the annual incidence of thyroid cancer has increased from 4.9-14.3 per 100,000.11 This rise in thyroid cancer has also occurred within Australia. The Cancer Council Victoria's 2015 Statistics and Trends report demonstrates an increasing incidence annual incidence of thyroid cancer from approximately $1-4.2$ per 100,000 men and 2.2-12 per 100,000 women. ${ }^{12}$ This is primarily attributable to increased detection via screening and improvements in resolution of imaging modalities such as ultrasound (US), computed tomography (CT), and positron-emission tomography/computed tomography (PET/CT). ${ }^{11}$ In South Korea, the incidence of thyroid cancer increased dramatically from 6.3 per 100,000 in 1999 to 47.5 per 100,00 in 2009 as a result of thyroid cancer screening initiatives. This resulted in an economic burden of thyroid cancer sevenfold, from $\$ 257$ million to $\$ 1724$ million, with no change in survival. ${ }^{13}$

$\mathrm{PET} / \mathrm{CT}$ is one particular imaging modality in which $\mathrm{TI}$ are being identified and increasingly studied. The enhanced uptake of glucose (or it's analogue FDG) by cancer cells due to inefficient aerobic glycolysis-termed the Warburg effect ${ }^{14}$-is the hallmark of in vivo cancer imaging with FDG PET/CT utilizing a radiotracer (FDG being the most common). Being a measure of the rate of intracellular glycolysis, concomitant benign, and malignant tumors unbeknown to the patient and treating physician may be detected. PET detected or "avid" "incidentalomas" have been reported to occur in up to $5 \%$ of scans.

As a consequence of increasing detection of thyroid cancer and the increasing use of PET/CT, incidentally detected thyroid nodules using this modality are becoming more apparent, and have been reported to occur in up to $2.5 \%$ of scans. ${ }^{1}$ The incidence of these "thyroid incidentalomas" (TI) varies widely within the literature, with a range from $0.02-8.9 \%$ from studies ranging from $689-15,711$ patients. ${ }^{1-3}$ Rates of malignancy varying also widely between 13 and $59 \%$ of $\mathrm{TI}^{2}{ }^{2} \mathrm{~A}$ large systematic review of the literature conducted by Nayan et al. totalling 197,296 PET studies was pooled from 31 studies, identified 3,659 (1.9\%) focal TI. ${ }^{3}$ In addition, a cross-sectional analysis conducted by Uppal et al. who reviewed 97,908 imaging studies demonstrated an incidence of benign and malignant TI to be $358 / 100,000$ PET scans. ${ }^{15}$ Incidental foci of FDG uptake unrelated to the primary malignancy therefore pose a significant problem for the reporting nuclear medicine specialist, treating physician and surgeon.

Our findings of a malignancy rate between 15 and $49.5 \%$ is consistent with the body of literature. An important limitation in determining an accurate malignancy rate across this and all retrospective studies are the absence of data from patients from patients who were not investigated with FNAC or surgery. Certainly it cannot be assumed that patients who were not investigated had benign thyroid nodules. Therefore we can only report an incidence "range" to reflect the probability of malignancy.

Observing patients with established cytological and histological diagnosed thyroid nodules, our data demonstrates that SUV-max does not reliably predict thyroid cancer, with an overall sensitivity of $70.9-73.4 \%$; specificity of $46.9-77.2 \%$; positive likelihood ratio of 3.12 and negative likelihood ratio of 0.38 . This is at an SUV-max cutoff of 5. The performance of SUV-max on ROC analysis was also poor, reflected by area under the curve (AUC) of 0.66 (whereby perfect separation of groups is reflected by an AUC of 1.0 and no separation of groups reflected by an AUC of 0.5) (Fig. 1). Therefore, the utility of an SUV-max cutoff to differentiate benign and malignant FDG-avid TI is currently not a useful method.

This finding is consistent with the large body of literature that SUV-max cutoffs are not a reliable means of predicting thyroid cancer. The largest sum of evidence published by Bertagna et al. demonstrates that approximately one half the number of studies investigating the utility of SUV-max show a statistically significant difference between benign and malignant nodules, whereas the other half show no difference. ${ }^{5}$ Bertagna also studied the power of SUV-max retrospectively across three nuclear medicine centers in a total of 211 patients (124 benign, 72 malignant, 4 nondiagnostic, and 11 indeterminate nodules) and demonstrated a very broad sensitivity of $57.1-95.7 \%$ and specificity of $46.4-79.3 \%$ across three centers at cutoffs of 4.8, 5.3, and 7. AUC ranged from 0.62-0.75, which confirmed the broad overlap of SUV-max between patients with benign and malignant $\mathrm{TI}^{1}$

A recent large single center study conducted by Yoon et al combining the Thyroid Imaging and Reporting Data System (TIRADS) scoring on ultrasound with SUV demonstrated that combined these tests improved sensitivity and negative predictive value, compared to SUV alone (97.5\% and $95 \%$, compared to $65 \%$ and $70 \%) .{ }^{16}$ However, 
when compared with TIRADS alone, the specificity and positive predictive value of the combined tests was reduced. The AUC on ROC analysis demonstrated an overall reduced performance of the combined test compared to TIRADS alone $(0.724$ and 0.737 , respectively). ${ }^{16}$ This demonstrates that SUV-max should also not be used as an adjunctive tool to increase the accuracy preexisting validated tools for thyroid cancer risk stratification.

The use of 18-FDG PET/CT has also been used to help define thyroid nodules that are repeatedly indeterminate or non-diagnostic on FNAC. This is a similar approach to investigating FDG-avid TI which do not have a definitive benign or malignant diagnosis with SUV-max. A systematic review and meta-analysis conducted by Vriens et al. in order to assess the pre-operative value of PET/CT and whether negative 18-FDG PET/CT can select patients who have a low suspicion of malignancy and avoid surgery. ${ }^{17}$ This study demonstrated that PET/CT had varying accuracy in diagnosing malignancy between $41.7 \%$ and $79 \%$ amongst six studies with small sample sizes ranging from 15-51 patients, with most false positive nodules were less than $15 \mathrm{~mm}$. The greatest impact from this study was the finding that a negative PET/CT (defined as no uptake) in nodules $>15 \mathrm{~mm}$ which had a low false negative rate of 3.6\%. ${ }^{17}$ The comparison of this data against TI demonstrates $\mathrm{PET} / \mathrm{CT}$ remains to be not a useful measure of malignancy amongst FDG-avid nodules. A negative PET/CT as defined by Vriens et al. may provide some reassurance to patients with repeatedly indeterminate FNAC, but unfortunately provides no reassurance to patients, endocrinologists, nuclear medicine physicians, and surgeons when encountered with FDG-avid TI.

SUV-max of FDG-avid TI is not usually reported by nuclear medicine physicians, as experienced within our institution. It is the wording of the report itself that guides a cancer clinician to whether the patient should be further investigated with ultrasound and FNAC. The excellent 5-year survival of PTC (greater than $90 \%$ ) needs to be balanced with the patient's stage of primary malignancy, their response to treatment and comorbidities prior to proceeding to biopsy. ${ }^{18}$

Therefore, utility of quoting an "incidence range" in PET/CT reports to risk stratify and aid decision making in respect to further investigation and treatment of FDG-avid TI may be limited. The key questions that arise following PET/CT reporting are "which patients should be further investigated with biopsy," and "if they are biopsied is surgical resection if a cancer is found appropriate in the context of the primary malignancy." This perspective is supported by Patterson et al. who found that FDG-avid TI had a minimal impact on overall and disease-specific survival in a large cohort of patients with nonthyroidal malignancy and the exceptionally low incidence of persistent clinically significant thyroid cancer in this series confirms that this decision is often made appropriately. ${ }^{19}$ The threshold for investigating suspicious lesions in the context of a primary nonthyroidal malignancy should depend on the prognosis of the primary malignancy.

There are other confounders of highly avid thyroid nodules which contribute to the broad overlap between benign and malignant TI. Hürthle cell nodules and follicular adenomas compared to other benign conditions. The association with high SUV in Hürthle cell nodules owes to impairment of respiratory chain complexes and consequent upregulation of oxidative phosphorylation and high mitochondrial density. ${ }^{20-22}$ Technical factors that can also contribute to varying SUV include level of fasting serum glucose, length of fasting period prior to examination, volume of FDG-injected, time of radiotracer administration, and uptake. ${ }^{5}$ In this study however, these factors were standardized by measuring the SUV-mean of the liver as quality assurance to ensure equal FDG uptake between studies.

\section{Conclusion}

As FDG-avid TI can demonstrate variable uptake of FDG with an overall sensitivity of $70.9-73.4 \%$; and specificity of $46.9-77.2 \%$, SUV-max is not a safe tool to discriminate benign from malignant TI. Our study therefore helps validate Bertagna's findings that SUV-max is not a safe tool to discriminate benign from malignant $\mathrm{TI}$.

\section{Clinical Significance}

SUV-max should remain a theoretical adjunctive tool for predicting thyroid cancer. The utility of quoting an incidence of thyroid cancer in PET/CT reports to risk stratify and aid decision making in respect to further investigation and treatment of FDG-avid TI may be limited. The threshold for investigating suspicious lesions with ultrasound and FNAC (in the context of primary nonthyroidal malignancy) should be determined by the prognosis of the primary malignancy.

\section{References}

1. Bertagna F, Treglia G, Piccardo A, et al. F18-FDG-PET/CT thyroid incidentalomas: a wide retrospective analysis in three Italian centres on the significance of focal uptake and SUV value. Endocrine 2013;43(3):678-685. DOI: 10.1007/s12020-012-9837-2

2. Gavriel H, Tang A, Eviatar E, et al. Unfolding the role of PET FDG scan in the management of thyroid incidentaloma in cancer patients. Eur Arch Otorhinolaryngol 2015;272(7):1763-1768. DOI: 10.1007/s00405-014-3120-5

3. Nayan S, Ramakrishna J, Gupta MK. The proportion of malignancy in incidental thyroid lesions on 18-FDG PET study: a systematic review and meta-analysis. Otolaryngol Head Neck Surg 2014;151(2):190-200. DOI: 10.1177/0194599814530861

4. Piccardo A, Puntoni M, Bertagna F, et al. 18F-FDG uptake as a prognostic variable in primary differentiated thyroid cancer incidentally detected by PET/CT: a multicentre study. Eur J Nucl Med Mol Imaging 2014;41(8):1482-1491. DOI: 10.1007/s00259-014-2774-y

5. Bertagna F, Treglia G, Piccardo A, et al. Diagnostic and clinical significance of F-18-FDG-PET/CT thyroid incidentalomas. J Clin Endocrinol Metab 2012;97(11):3866-3875. DOI: 10.12 10/jc.2012-2390

6. Shie P, Cardarelli R, Sprawls K, et al. Systematic review: prevalence of malignant incidental thyroid nodules identified on fluorine-18 fluorodeoxyglucose positron emission tomography. Nucl Med Commun2009;30(9):742-748. DOI: 10.1097/MNM.0b013e32832ee09d

7. Abraham T, Schöder H. Thyroid cancer-indications and opportunities for positron emission tomography/computed tomography imaging. Semin Nucl Med 2011;41(2):121-138. DOI: 10.1053/j.semnuclmed.2010.10.006

8. Pampaloni MH, Win AZ. Prevalence and characteristics of incidentalomas discovered by whole body FDG PETCT. Int J Mol Imaging 2012;2012:476763. DOI: 10.1155/2012/476763

9. Cibas ES, Ali SZ. The Bethesda System for reporting thyroid cytopathology. Thyroid 2009;27(11):1341-1346. DOI: 10.1089/thy.2017.0500

10. Lloyd RV, Osamura RY, Klöppel G, et al. WHO classification of tumors, pathology and genetics-tumors of endocrine organstle. IARC Press Lyon. 2004; ISBN: 978-92-832-4493-6.

11. Haugen BR, Alexander EK, Bible KC, et al. 2015 American Thyroid Association Management Guidelines for adult patients with thyroid nodules and differentiated thyroid cancer: The American Thyroid Association Guidelines task force on thyroid nodules and differentiated thyroid cancer. Thyroid 2016;26(1):1-133. DOI: 10.1089/thy.2015.0020 
12. Thursfield V, Farrugia H. Cancer in Victoria: statistics and trends 2015. Cancer Counc Victoria 2015;1-63.

13. Park S, Oh C-M, Cho H, et al. Association between screening and the thyroid cancer "epidemic" in South Korea: evidence from a nationwide study. BMJ 2016;(355):5745. DOI:10.1136/bmj.i5745

14. Warburg O. On the origin of cancer cells. Science 1956;123(3191): 309-314. DOI: 10.1126/science.123.3191.309

15. Uppal A, White MG, Nagar S, et al. Benign and malignant thyroid incidentalomas are rare in routine clinical practice: a review of 97,908 imaging studies. Cancer Epidemiol Biomarkers Prev 2015;24(9):1327-1331. DOI: 10.1158/1055-9965.EPI-15-0292

16. Yoon JH, Cho A, Lee HS, et al. Thyroid incidentalomas detected on 18F-fluorodeoxyglucose-positron emission tomography/computed tomography: Thyroid Imaging Reporting and Data System (TIRADS) in the diagnosis and management of patients. Surgery 2015;158(5):1314-1322. DOI: 10.1016/j.surg.2015.03.017

17. Vriens D, De Wilt JHW, Van Der Wilt GJ, et al. The role of [18F]2-fluoro-2-deoxy-d-glucose-positron emission tomography in thyroid nodules with indeterminate fine-needle aspiration biopsy: Systematic review and meta-analysis of the literature. Cancer 2011;117:4582-4594. DOI: 10.1002/cncr.26085
18. Edge SB, Compton CC. The American Joint Committee on Cancer: the 7th edition of the AJCC cancer staging manual and the future of TNM. Ann Surg Oncol 2010;17(6):1471-1474. DOI: 10.1245/s10434-010-0985-4

19. Pattison DA, Bozin M, Gorelik A, et al. 18 F-fluorodeoxyglycose-avid thyroid incidentalomas: the importance of contextual interpretation. J Nucl Med 2018;59(5):749-755. DOI:10.2967/jnumed.117.198085

20. Pattison D, Angel C, Bozin M, et al. Oncocytic thyroid nodules are a common aetiology for intensely $18 \mathrm{~F}$-fluorodeoxyglucose-avid thyroid incidentalomase. Thyroid 2015;25(S1):337. DOI: http://doi. org/10.1089/thy.2015.29004.abstracts

21. Goffredo P, Roman SA, Sosa JA. Hurthle cell carcinoma: a population-level analysis of 3311 patients. Cancer 2013;119(3):504-511. DOI: $10.1002 / \mathrm{cncr} .27770$

22. Máximo V, Lima J, Prazeres $\mathrm{H}$, et al. The biology and the genetics of Hurthle cell tumors of the thyroid. Endocr Relat Cancer 2012;19(4):131-147. DOI: 10.1530/ERC-11-0354 